\title{
A Smart Reporting System for Determining the Physiological State and Body Posture of Elderly People
}

\author{
Shun-Peng Shih * Wei-Der Chang Tien-Yu Liang Hao-Tse Huang Yi-Ting Hsiao Yu-Ting Xia \\ Department of Computer and Communication, Shu-Te University \\ Kaohsiung 824, Taiwan
}

*Corresponding Author: spshih@stu.edu.tw

\begin{abstract}
In this paper, a device was designed and developed by integrating ZigBee technology with radio-frequency identification technology, a triaxial accelerometer, a sphygmomanometer, an Android application, and a neural network algorithm. The CC2530 system chips built using the ZigBee technology were combined with a triaxial accelerometer and a back-propagation neural network algorithm was added to improve the accuracy of the neural network, enabling accurate assessment for determining whether incidents such as falling have occurred. The active radio-frequency identification technology model and the sphygmomanometer were employed to determine the physiological state of users. By using a Bluetooth module and a smartphone, text messages were sent to family members or caregivers when accidents, such as falling and abnormal body temperature, occurred, validating the effectiveness of the designed device as a smart physiological state and body posture reporting system.
\end{abstract}

Keywords: ZigBee, triaxial accelerometer, home care.

\section{Study Motivation}

Advances in technology and medical care have generated numerous resources for the public. However, contemporary societies face problems related to population ageing because of low birth rates. In addition, because a majority of young workers are employed in locations away from home, they are unable to care for their elders on a regular basis. These elderly people thus live alone and remain uncared for. Therefore, an idea to equip elderly people with sensors is proposed. These sensors can measure the blood pressure and pulse of elderly people, enabling them to measure these values at their own homes, and the system can automatically transmit the data to a computer for analysis. When these elderly people accidentally fall or get into incidents, the sensors detect the signals and forward the information to the system. After determining the type of incident that had occurred, the system immediately transmits a text message to the mobile phone of selected family members or caregivers, enabling them to instantly know of the elderly person's situation. Contingency plans were also established for accidents, and the global positioning system (GPS) function was activated when elderly people left their residences. This enabled others to immediately identify the location of elderly people.

Advances in medical treatment and society have engendered an increase in the average life expectancy and the number of elderly people aged 65 or above, which increased from 1.9 million people in 2000 (yearend) to 2.5 million in 2010. Coupled with the declining birth rate, the population ageing situation has become increasingly pronounced.

When elderly people who live alone or those whose family members have gone to work fall, they are immobilized and may even become unconscious. If these elderly people are left unattended for a prolonged period, the elderly people may be in severe danger and may even die. Therefore, a system was designed for determining the type of incident that has occurred before sending a text message to notify selected family members or caregivers for help.

\section{System Framework}


As shown in Fig. 1, a smart physiological state and body posture reporting system for elderly people was developed in this study. A wireless sensor network using ZigBee technology ${ }^{(6)}$ was built; the technology featured short-distance wireless communication capability and added triaxial acceleration sensors (ADXL345 ${ }^{(13)}$ ) to the network end devices. $\mathrm{CC} 2530^{(14)}$ chips were used to obtain the three-axis motion data, where the neural network was adopted as the core algorithm used to create a human-posture recognition system. The resulting device was then equipped on an appropriate location of the users' body where signals were transmitted to the ZigBee coordinator before being transmitted to the computer through serial universal asynchronous receivers/transmitters. The device has indoor and outdoor functions. In an indoor environment, the device was used with the radio-frequency identification (RFID) system $^{(15)}$ to detect whether users were within range. The active tag could be used to measure the body temperature and identify when abnormal body temperature occurred. Moreover, a sphygmomanometer was set in place to measure the blood pressure and pulse of users. In an outdoor environment, a Bluetooth system was used to resend the information to the mobile phone, enabling the GPS function to identify the users' current location. When accidents occur, the system sends a text message to selected family members or caregivers.

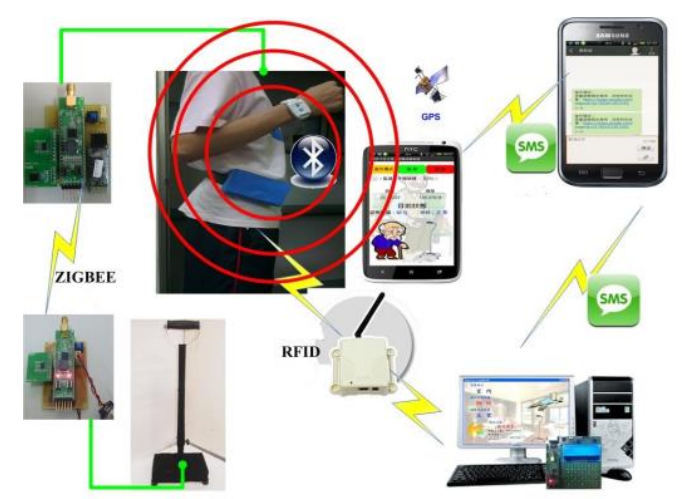

Fig. 1. System framework.

\section{Implementation Process and Results}

Regarding the designed device, the body posture reporting system used the triaxial accelerometer and was integrated with a ZigBee technology called MCU-CC2530 chips (Fig. 2). In addition, the back-propagation neural network algorithm was adopted to improve the range of applicability of the device.

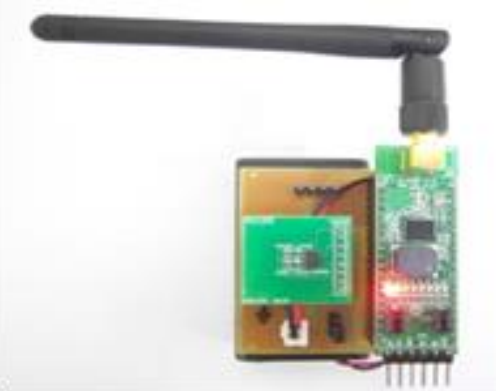

Fig. 2. The triaxial accelerometer combined with the CC2530 chips.

Relationship between the output value and the input value of Processing unit for neural network could be generally represented by using the sum of product of weighting and input values, equation expressed as equation (1), where $\mathrm{Y}_{\mathrm{j}}$ is output signal of model, $\mathrm{W}_{\mathrm{ij}}$ is weighting value, $\mathrm{Xi}$ is input signal, $\theta \mathrm{j}$ is the threshold value. The back-propagation neural network model is currently the most representative and common model among all neural network models ${ }^{(9)}$. Moreover, it is a supervised learning network, which is divided into two stages comprising network training and network recall.

$$
Y_{j}=f\left(\sum_{i} W_{i j} X_{i}-\theta_{j}\right),
$$

The process, which begins from tests to simulations and to device completion was divided into three steps. The first step involved designing a program to test and receive the triaxial data, where the $\mathrm{CC} 2530$ chips were used for the ZigBee wireless network communication. The device transferred triaxial data to the coordinator in the form of packets and a program written using VB.NET program was used to verify whether that the packets were received in full. The data then underwent numerical normalization and were used for the network training part of the algorithm ${ }^{(1,2,3)}$.

The second step involved developing a back-propagation neural network training program where the network training program was independently written and each calculation procedure in the algorithm was executed independently, the program interface is shown as Fig. 3. 


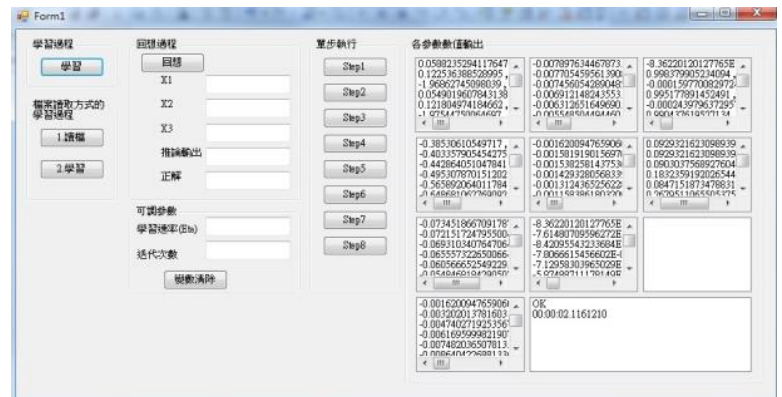

Fig. 3. The program interface of the learning algorithm during the writing stage.

The back-propagation network training program confirmed whether each parameter was correct and repeatedly corrected the numerical values, read the normalized triaxial data, designed the action codes, and performed network training until the algorithm was confirmed to be effective for converging network errors (Fig. 4). The model flow and setting value of the initial weights $\mathbf{W}$, thresholds $\theta$ and rates of learning $\eta$ in this model is indicated as Fig. 5.

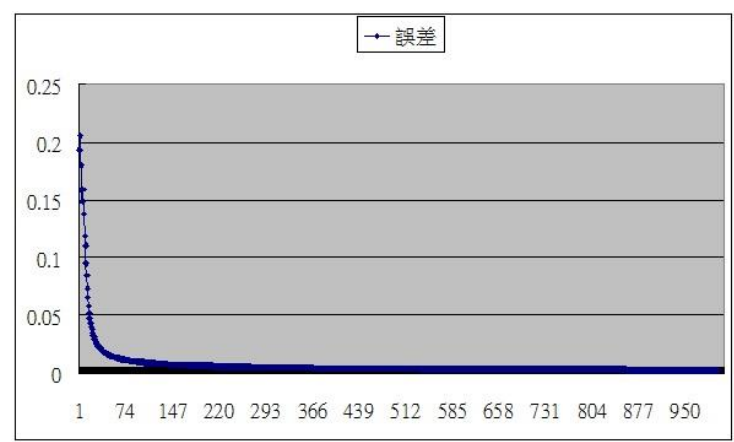

Fig. 4. Error convergence (every 1,000 network training sessions).

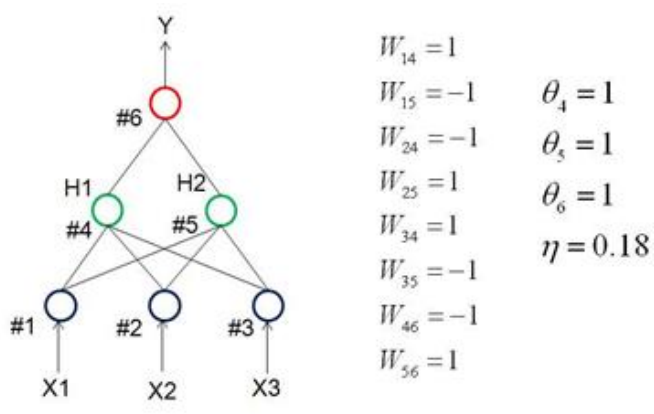

Fig. 5. The model flow and setting value of the initial weights, thresholds and rates of learning in this model.

The third step involved a program for sending network recalls and judging user movements. The ZigBee devices featuring triaxial functions were equipped near the waist of the users (Fig. 6) to determine movement.

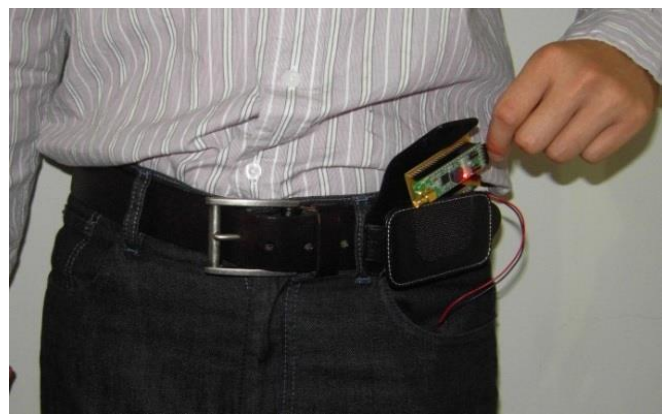

Fig. 6. The location where the ZigBee device was equipped.

Upon receiving the information from the device, the program immediately performed numerical normalizations on the data, which were then used as the network feedback information. Calculations of inferred outputs were made, enabling immediate posture identification (Fig. 7).

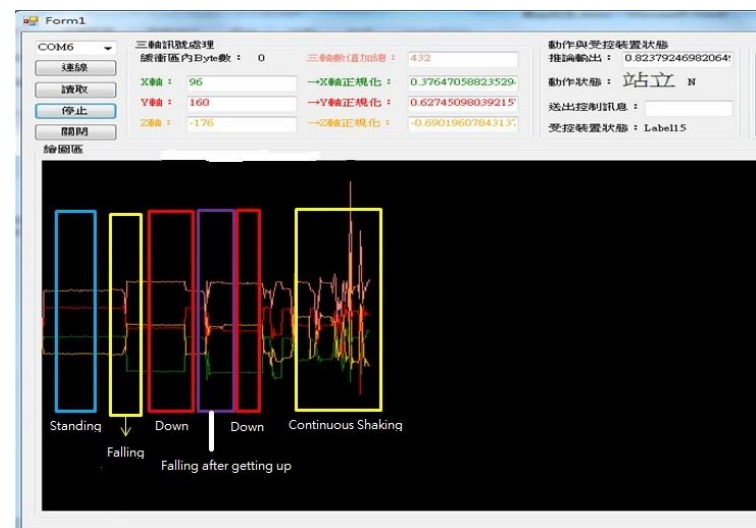

Fig. 7. Instant identification of continuous movements from standing $\rightarrow$ falling $\rightarrow$ getting up $\rightarrow$ falling.

We totally carried out 30 times experiments for each posture movement, the result shows recognition rate is exceed $90 \%$ in table 1.

Table. 1. Experiments for each direction movement.

\begin{tabular}{|c|c|c|c|c|}
\hline Result Direction & Forward & Back & Left & Right \\
\hline Falling to down & 29(times) & 27 & 28 & 28 \\
\hline Unrecognized & 0 (times) & 0 & 0 & 0 \\
\hline Recognition rate & $97 \%$ & $90 \%$ & $93 \%$ & $93 \%$ \\
\hline
\end{tabular}

In addition to the aforementioned body posture reporting system, this device features a physiological state 
display, which measures the physiological information of the users such as blood pressure and body temperature. A blood pressure measurement module was added on the device for daily measurements and to record systolic and diastolic blood pressure (Fig. 8); the information was sent to the proposed device in packets, allowing it to determine whether the values fulfilled health standards.

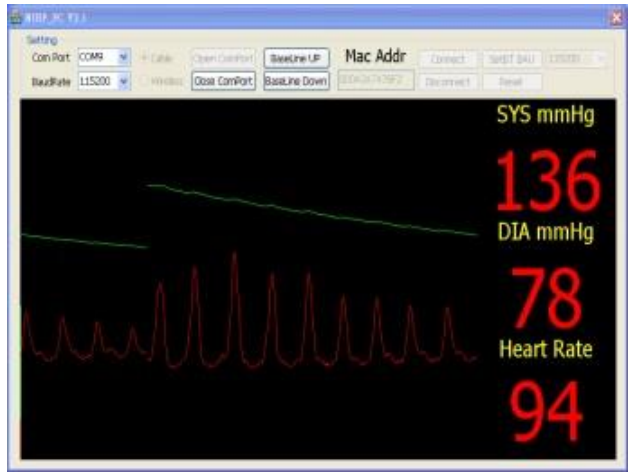

Fig. 8. Original program used for blood pressure measurement.

The RFID module was used to collect body temperature data. The users wore a wrist tag that contained a temperature sensor. The tag's packet information included parameters such as a unique identification number, received signal strength indication, and body temperature. The active RFID reader located in a fixed location in the room assessed the unique identication number of the received signal strength indication in the default range to confirm whether users were within range and if their body temperatures were normal. It actively checked the health and safety of the users, preventing them from being lost or from physical discomforts such as fever. After completing the test phase for all the functions, the device was practically applied. The users were equipped with active RFID $^{(15)}$ tags on their wrists and ZigBee devices on their waists (Fig. 9).

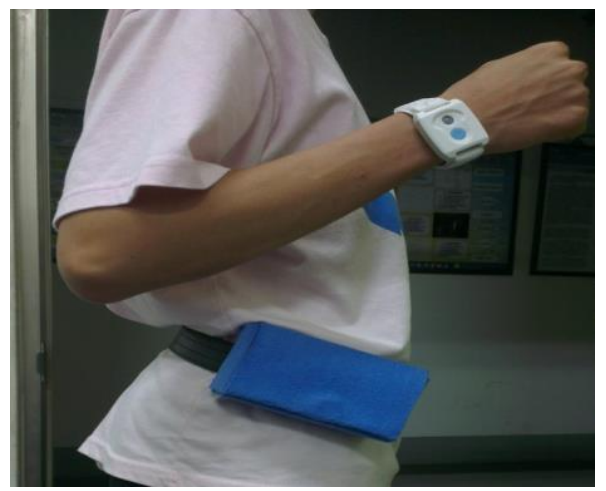

Fig. 9. Schematic diagram of user with an active RFID tag and a ZigBee device.
The ZigBee device electric circuit comprised CC2530 chips, a triaxial accelerometer, a vibration sensor, and a Bluetooth system shown as Fig. 10. It was equipped around the waist of users. In the future, the researchers will consider miniaturizing the electric circuit to make the device more convenient for users to wear.

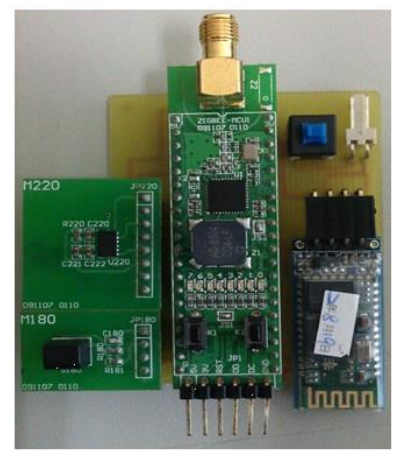

Fig. 10. The ZigBee device.

Because the target users were primarily elderly people, most of whom had difficulty walking and required the use of a walking stick, a modified version of the device that can be used with a walking stick was designed. The walking sticks used in this study were equipped with a light-emitting diode to provide lighting at night. A ZigBee device was placed at the base of the walking sticks, which were also equipped with a triaxial accelerometer for determining whether the sticks had dropped to the ground to deduce if the elderly people had fallen. In addition, a buzzer was added to attract the attention of passersby. Because walking sticks are inanimate objects and instances of users dropping their sticks without actually falling may be possible, a $10 \mathrm{~s}$ buffer time for false alarms was included in the ZigBee device.

The device has indoor and outdoor functions. When the device mode is set as indoor, caregivers can be aware whether users are currently safe by referring to the interface, which provides user information such as posture, walking stick condition, blood pressure, and body temperature. Combined with the text messaging function of mobile phones, messages can be sent to the phones of caregivers when users fall, experience abnormal body temperatures, or move beyond a designated area; this enables caregivers to immediately learn of the users' situation and provide timely assistance. The Main program interface for user is shown as Fig. 11 


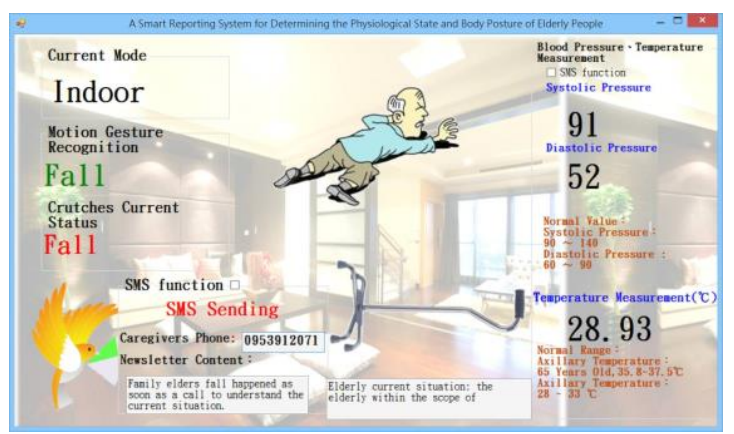

Fig. 11. Main program interface (when users have fallen)

Conversely, when the device mode is set as outdoor, the CC2530 chips automatically determine when users fall and notifications are sent through the Bluetooth system to a smartphone. The app ${ }^{(4,5)}$ shows the users' current condition and the GPS coordinates. When the CC2530 chips determine that users have fallen, signals are sent to the mobile phone and the app detects the signals (Fig. 12) and immediately sends a text message to the mobile phone of the caregivers.

When a distress message is received (Fig. 13), caregivers are able to know about the user's situation by reading the message and their GPS coordinates. Caregivers merely have to click on the GPS coordinates hyperlink to activate the Google Maps function and identify the user's exact location, which enables them to immediately locate the user in outdoor environments.

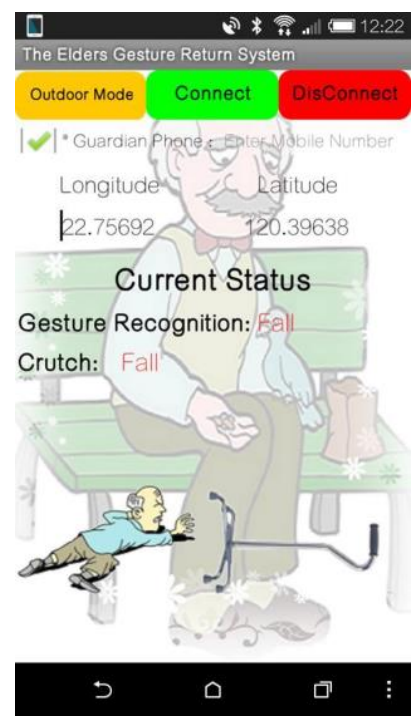

Fig. 12. The Android program interface (when users have fallen).

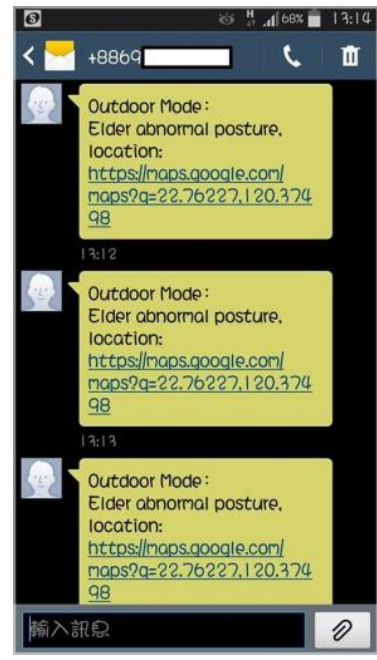

Fig. 13. Distress message.

\section{Conclusion}

The Internet of Things is a crucial indicator of the development of science and technology. As wireless communications technology increasingly matures, a network connecting all things will not only become a reality, but also serve as an integral technology in contemporary lifestyles. Smartphones, computers, and tablet computers can be considered different "domains" connected to the same network, which is the Internet. In the online world, these different platforms will foster additional smart applications. Some of the functions provided by the device developed in this study, such as posture identification and health management, have already been used in a number of prototypes and products. The proposed smart physiological state and body posture reporting system, designed for elderly people who live alone or in nursing homes, combines the two aforementioned functions to facilitate immediate notifications and actions when elderly people fall. Because this device can also be used to monitor the users' physiological condition on a daily basis, its presentation is expected to lead to future usage for improving the care that elderly people receive in Taiwan.

\section{Acknowledgment}

This work was supported by the Ministry of Science and Technology of Taiwan under the grant MOST 104-2221-E-366-005.

\section{References}

(1) Tu K.H., Shi S.P., Huang Z.Y., and Sun W.L. (2011). 
The Use of Neural Networks in Human Body Posture Recognition. Shu-Te University Department of Computer and Communication, 2010 Symposium on Applications of Information, Management and Communication Technology.

(2) Tu K.H. (2011). The Recognition and Application of the Real-Time Human Body Motion based on Artificial Neural Network. Master's Thesis, Shu-Te University.

(3) Tu K.H. (2010). Human Body Posture Recognition Systems. Shu-Te University, 2010 National Symposium on Telecommunications.

(4) Zeng J.H., Cai Y.T., Huang K.Q., Lai W.M.., Lu W.Y, and Shi L.W. (2012). A Beginner's Guide to Android App "App Inventor." Fullon Publishing.

(5) Zeng J.H., Lai W.M., Xie Z.H., Lin Y.X., and Xue H.Y. (2012). A Beginner's Guide to Android App, the "App Inventor Robots." Fullon Publishing.

(6) Zheng L. (2008). System Development Using the ZigBee. Chuan Hwa Book Co., Ltd., Taipei.

(7) Huang Z.Y. (2011). The Recognition and Application of the Real-Time Human Falls based on Artificial Neural Network Shu-Te University, 2011 National Symposium on Telecommunications.

(8) Ye Y.C. (1997). The Application of Neural Networks. Scholars Books.

(9) Ye Y.C. (1999). The Application and Implementation of Neural Network Models. Scholars Books.

(10)Zeng Y.Q., Pan M.X., and Lin Z.Y. (2006). Wireless and Local Area Network: The Technology and Applications of Casual and Sensor Network, Advisory Council, Ministry of Education.

(11) Chen L.Y., Fan Y.Z., and Liao J.Q. (2006). Visual Basic 2005 and Automated System Monitoring. Unalis Co. Ltd.

(12) Xu Q.R. and Mai D.C. (2006). The Ultimate Guide to Visual Basic 2005 Express Program Design. DrMaster Press Co., Ltd., Taipei.

(13) ADXL345 Datasheet, www.analog.com。

(14) CC2530 (Rev. B), www.ti.com

(15)Liao G.L., Lin J.Z., Xu Y.H., and Li J.F. (2010). RFID Radio Frequency Identification Experimental System. Taiwan Ubiquitous Information Co., Ltd., Kaohsiung. 